DOI: $10.15393 / \mathrm{j} 3$.art.2019.6290

UDC 517.587, 517.521.1

B. Aloui, L. KhÉRIJI

\title{
CONNECTION FORMULAS AND REPRESENTATIONS OF LAGUERRE POLYNOMIALS IN TERMS OF THE ACTION OF LINEAR DIFFERENTIAL OPERATORS
}

\begin{abstract}
In this paper, we introduce the notion of $\mathfrak{O}_{\varepsilon}$-classical orthogonal polynomials, where $\mathfrak{O}_{\varepsilon}:=\mathbb{I}+\varepsilon D(\varepsilon \neq 0)$. It is shown that the scaled Laguerre polynomial sequence $\left\{a^{-n} L_{n}^{(\alpha)}(a x)\right\}_{n \geqslant 0}$, where $a=-\varepsilon^{-1}$, is actually the only $\mathfrak{O}_{\varepsilon}$-classical sequence. As an illustration, we deal with some representations of Laguerre polynomials $L_{n}^{(0)}(x)$ in terms of the action of linear differential operators on the Laguerre polynomials $L_{n}^{(m)}(x)$. The inverse connection problem of expanding Laguerre polynomials $L_{n}^{(m)}(x)$ in terms of $L_{n}^{(0)}(x)$ is also considered. Furthermore, some connection formulas between the monomial basis $\left\{x^{n}\right\}_{n \geqslant 0}$ and the shifted Laguerre basis $\left\{L_{n}^{(m)}(x+1)\right\}_{n \geqslant 0}$ are deduced.
\end{abstract}

Key words: Classical polynomials, Laguerre polynomials, lowering and raising operators, structure relations, higher order differential operators, connection formulas

2010 Mathematical Subject Classification: 33C45, 42C05

1. Introduction. Let $\mathcal{O}$ be a linear operator that acts on the space $\mathcal{P}$ of polynomials in one variable and maps polynomials of degree $n$ to polynomials of degree $n+n_{0}$ ( $n_{0}$ is a fixed integer $)$. We call a sequence $\left\{p_{n}\right\}_{n \geqslant 0}$ of orthogonal polynomials $\mathcal{O}$-classical if there exist a sequence $\left\{q_{n}\right\}_{n \geqslant 0}$ of orthogonal polynomials such that $\mathcal{O} p_{n}=q_{n+n_{0}}$, where $n \geqslant 0$ if $n_{0} \geqslant 0$ and $n \geqslant n_{0}$ if $n_{0}<0$. (This is Hahn's property [1-4], [6-8], [12], [13], [15], [18], [19], [23]).

It is known that the monic Laguerre polynomial sequence $\left\{L_{n}^{(\alpha)}\right\}_{n \geqslant 0}$, where $\alpha \neq-n, n \geqslant 1$, is classical and satisfies the relation (see [10], [16])

$$
D L_{n}^{(\alpha)}=n L_{n-1}^{(\alpha+1)}, \quad n \geqslant 1 .
$$

(C) Petrozavodsk State University, 2019 
In [2], the first author introduced the notion of $\mathcal{R}_{\alpha}$-classical orthogonal polynomials and put in evidence, for $\alpha \in \mathbb{C} \backslash\{0,-1,-2, \ldots\}$, the following relation

$$
\mathcal{R}_{\alpha} L_{n}^{(\alpha)}(x)=L_{n+1}^{(\alpha-1)}(x), \quad n \geqslant 0 .
$$

Note that whereas the first expression involves the operator $D$ that lowers the degree and raises the parameters, the second one involves $\mathcal{R}_{\alpha}:=(x-\alpha) \mathbb{I}-x D$, which raises the degree and lowers the parameters. The two operators together are called shift operators. In [19], the authors proved that $\left\{L_{n}^{(\alpha)}\right\}_{n \geqslant 0}$ is the only $\mathrm{F}_{\alpha}$-Appell orthogonal sequence and satisfies

$$
\mathrm{F}_{\alpha} L_{n}^{(\alpha)}(x)=c_{n} L_{n-1}^{(\alpha)}(x), \quad n \geqslant 0,
$$

where $\mathrm{F}_{\alpha}:=D x D+\alpha D, \alpha \in \mathbb{C} \backslash\{-1,-2, \ldots\}$, is called lowering operator (it lowers the degree and preserves the parameter) introduced by Dattoli and Ricci (see [11]). We also see, by the second order differential equation satisfied by the Laguerre polynomials, that [9]

$$
\mathcal{L} L_{n}^{(\alpha)}(x)=\lambda_{n} L_{n}^{(\alpha)}(x), \quad n \geqslant 0,
$$

where $\mathcal{L}:=x D^{2}-(x-\alpha-1) D$, is called Jacobi's operator. Note that the classical orthogonal polynomials (Hermite, Laguerre, Bessel, and Jacobi) are essentially the only eigenfunctions of the Bochner's operator, i. e., satisfy the same relation (4) (see [9]).

Furthermore, the present contribution is a natural continuation of a previous works. More precisely, in view of Eqs (1)-(4), it is natural to study the same problem with respect to the operator which, for example, raises the parameters and preserves the degree of the polynomial $L_{n}^{(\alpha)}(x), n \geqslant 0$. The operator is $\mathfrak{O}_{\varepsilon}:=\mathbb{I}+\varepsilon D(\varepsilon \neq 0)$. The basic idea has been deduced by starting from the so called second structure relation $[20,21]$

$$
L_{n}^{(\alpha)}(x)=(n+1)^{-1} L_{n+1}^{(\alpha)^{\prime}}(x)+L_{n}^{(\alpha)^{\prime}}(x), n \geqslant 0,
$$

which gives, by using (1), the following relation

$$
\mathfrak{O} L_{n}^{(\alpha)}(x)=L_{n}^{(\alpha+1)}(x), \quad n \geqslant 0,
$$

where $\mathfrak{O}:=\mathbb{I}-D$, with $\mathbb{I}$ as the identity operator. This means that the above family of standard orthogonal polynomials is an $\mathfrak{O}$-classical polynomial sequence with respect to the operator $\mathfrak{O}$, i. e., it is an orthogonal 
polynomial sequence, whose sequence of $\mathfrak{O}$ is also orthogonal. For a given $\varepsilon \neq 0$, let us consider $\mathfrak{O}_{\varepsilon}: \mathcal{P} \rightarrow \mathcal{P}$ the linear operator defined in the linear space $\mathcal{P}$ of polynomials with complex coefficients

$$
\mathfrak{O}_{\varepsilon}:=\mathbb{I}+\varepsilon D \quad\left(\mathfrak{O}_{-1}=\mathfrak{O}\right) .
$$

The aim of this paper is to put in evidence the relation (5) and characterize the $\mathfrak{O}_{\varepsilon}$-classical orthogonal polynomials.

The further contents of this paper is as follows. Section 2 gives some preliminaries, while the main result is proved in Section 3. In Sections 4 and 5 , we give some new properties related to the above operator and the Laguerre polynomials.

2. Preliminaries. Let $\mathcal{P}$ be the linear space of polynomials in one variable with complex coefficients. Let $\mathcal{P}^{\prime}$ be the algebraic linear dual of $\mathcal{P}$. We write $\langle u, p\rangle:=u(p)\left(u \in \mathcal{P}^{\prime}, p \in \mathcal{P}\right)$. A linear functional $u \in \mathcal{P}^{\prime}$ is said to be regular or quasi-definite [10], [22] if $\operatorname{det}\left\langle u, x^{i+j}\right\rangle_{i, j=1, \ldots, n} \neq 0$ for $n \geqslant 0$. This is equivalent to the existence of a unique sequence of monic polynomials $\left\{P_{n}\right\}_{n \geqslant 0}$ of degree $n$ such that $\left\langle u, P_{n} P_{m}\right\rangle=r_{n} \delta_{n, m}, n, m \geqslant 0$, with $r_{n} \neq 0(n \geqslant 0)$. The sequence $\left\{P_{n}\right\}_{n \geqslant 0}$ is then called a monic orthogonal polynomial sequence (MOPS) with respect to $u$.

Theorem 1. (Favard's Theorem [10]). Let $\left\{P_{n}\right\}_{n \geqslant 0}$ be a monic polynomial sequence. Then $\left\{P_{n}\right\}_{n \geqslant 0}$ is orthogonal if and only if there exist two sequences of complex numbers $\left\{\beta_{n}\right\}_{n \geqslant 0}$ and $\left\{\gamma_{n}\right\}_{n \geqslant 0}$, such that $\gamma_{n} \neq 0, n \geqslant 1$ and satisfies the three-term recurrence relation

$$
(\mathrm{TTRR})\left\{\begin{array}{l}
P_{0}(x)=1, P_{1}(x)=x-\beta_{0}, \\
P_{n+2}(x)=\left(x-\beta_{n+1}\right) P_{n+1}(x)-\gamma_{n+1} P_{n}(x), n \geqslant 0 .
\end{array}\right.
$$

When $\left\{P_{n}\right\}_{n \geqslant 0}$ is a MOPS, then $\left\{\tilde{P}_{n}\right\}_{n \geqslant 0}$, where $\tilde{P}_{n}(x)=a^{-n} P_{n}(a x+$ $+b),(a, b) \in \mathbb{C}^{*} \times \mathbb{C}$, is also a MOPS and satisfies [20], [21]

$$
\left\{\begin{array}{l}
\tilde{P}_{0}(x)=1, \quad \tilde{P}_{1}(x)=x-\tilde{\beta}_{0}, \\
\tilde{P}_{n+2}(x)=\left(x-\tilde{\beta}_{n+1}\right) \tilde{P}_{n+1}(x)-\tilde{\gamma}_{n+1} \tilde{P}_{n}(x), n \geqslant 0
\end{array}\right.
$$

where $\tilde{\beta}_{n}=a^{-1}\left(\beta_{n}-b\right)$ and $\tilde{\gamma}_{n+1}=a^{-2} \gamma_{n+1}$.

An orthogonal polynomial sequence $\left\{P_{n}\right\}_{n \geqslant 0}$ is called classical, if $\left\{P_{n}^{\prime}\right\}_{n \geqslant 0}$ is also orthogonal (Hermite, Laguerre, Bessel, or Jacobi), [10]. This is essentially the Hahn-Sonine characterization (see [12], [24]) of the classical orthogonal polynomials. 
It is well-known that any classical polynomial sequence $\left\{P_{n}\right\}_{n \geqslant 0}$ can be characterized taking into account its orthogonality as well as the First Structure Relation (FSR), or the Second Structure Relation (SSR) [5], [20], [21]:

(FSR) $\quad \phi(x) P_{n+1}^{\prime}(x)=r(x, n) P_{n+1}(x)+s_{n} P_{n}(x), n \geqslant 0$,

(SSR) $\quad P_{n}(x)=(n+1)^{-1} P_{n+1}^{\prime}(x)+a_{n} P_{n}^{\prime}(x)+b_{n} P_{n-1}^{\prime}(x), n \geqslant 0$.

Note that if $P_{n}(x)=L_{n}^{(\alpha)}(x),(\alpha \neq-n, n \geqslant 1)$ is the monic Laguerre polynomial, then we have a MOPS for which formulas (6), (8), and (9) were given for $n \geqslant 0$ by [16], [20], [22]

$$
(\mathrm{TTRR})\left\{\begin{array}{r}
L_{0}^{(\alpha)}(x)=1, \quad L_{1}^{(\alpha)}(x)=x-\alpha-1 \\
L_{n+2}^{(\alpha)}(x)=(x-(2 n+\alpha+1)) L_{n+1}^{(\alpha)}(x)- \\
\quad-(n+1)(n+\alpha+1) L_{n}^{(\alpha)}(x) .
\end{array}\right.
$$

$(\mathrm{FSR}) x L_{n+1}^{(\alpha)^{\prime}}(x)=(n+1) L_{n+1}^{(\alpha)}(x)+(n+1)(n+\alpha+1) L_{n}^{(\alpha)}(x), n \geqslant 0$,

$$
\text { (SSR) } L_{n}^{(\alpha)}=(n+1)^{-1} L_{n+1}^{(\alpha)^{\prime}}(x)+L_{n}^{(\alpha)^{\prime}}(x) n \geqslant 0 .
$$

Note that the monic Laguerre polynomial can be expressed by the Rodrigues formula [17]

$$
L_{n}^{(\alpha)}(x)=(-1)^{n} e^{x} x^{-\alpha} \frac{d^{n}}{d x^{n}}\left(e^{-x} x^{n+\alpha}\right), n \geqslant 0 .
$$

It also satisfies the following explicit representation [25]:

$$
L_{n}^{(\alpha)}(x)=\sum_{\nu=0}^{n}(-1)^{n-\nu}\left(\begin{array}{l}
n \\
\nu
\end{array}\right) \frac{\Gamma(n+\alpha+1)}{\Gamma(\nu+\alpha+1)} x^{\nu}, n \geqslant 0 .
$$

Recall the two formulas [25], [26]: for all $n \geqslant 0, \alpha>-1$,

$$
L_{n}^{(\alpha)}(t x)=\sum_{k=0}^{n}(-1)^{n-k}\left(\begin{array}{l}
n \\
k
\end{array}\right) \frac{\Gamma(n+\alpha+1)}{\Gamma(k+\alpha+1)} t^{k}(1-t)^{n-k} L_{k}^{(\alpha)}(x),
$$

and for all $n \geqslant 0, \alpha>-\frac{1}{2}$ :

$$
L_{n}^{(\alpha)}(x)=\frac{n ! \Gamma(n+\alpha+1)}{\sqrt{\pi}(2 n) ! \Gamma\left(\alpha+\frac{1}{2}\right)} \int_{-1}^{1}\left(1-t^{2}\right)^{\alpha-\frac{1}{2}} H_{2 n}(t \sqrt{x}) \mathrm{d} t
$$


where $H_{n}(x)$ is the Hermite polynomial on degree $n$.

\section{Hahn's property with respect to the operator $\mathfrak{O}_{\varepsilon}$.}

Recall that the operator $\mathfrak{O}_{\varepsilon}$ is defined by

$$
\begin{aligned}
\mathfrak{O}_{\varepsilon}: \mathcal{P} & \longrightarrow \mathcal{P} \\
f & \longmapsto f+\varepsilon f^{\prime},(\varepsilon \neq 0) .
\end{aligned}
$$

Our purpose here is to describe all the $\mathfrak{O}_{\varepsilon}$-classical orthogonal polynomials, i. e., the SMOP $\left\{P_{n}\right\}_{n \geqslant 0}$ such that the monic sequence $\left\{Q_{n}\right\}_{n \geqslant 0}$, where

$$
Q_{n}(x)=P_{n}(x)+\varepsilon P_{n}^{\prime}(x), n \geqslant 0,
$$

is also orthogonal. Suppose that $\left\{P_{n}\right\}_{n \geqslant 0}$ and $\left\{Q_{n}\right\}_{n \geqslant 0}$ are SMOP satisfying

$$
\begin{aligned}
& \left\{\begin{array}{l}
P_{0}(x)=1, P_{1}(x)=x-\beta_{0}, \\
P_{n+2}(x)=\left(x-\beta_{n+1}\right) P_{n+1}(x)-\gamma_{n+1} P_{n}(x), \gamma_{n+1} \neq 0, n \geqslant 0,
\end{array}\right. \\
& \left\{\begin{array}{l}
Q_{0}(x)=1, Q_{1}(x)=x-\chi_{0}, \\
Q_{n+2}(x)=\left(x-\chi_{n+1}\right) Q_{n+1}(x)-\theta_{n+1} Q_{n}(x), \theta_{n+1} \neq 0, n \geqslant 0 .
\end{array}\right.
\end{aligned}
$$

We have the following result.

Lemma 1. The sequences $\left\{P_{n}\right\}_{n \geqslant 0}$ and $\left\{Q_{n}\right\}_{n \geqslant 0}$ are related as follows:

$$
\begin{aligned}
& P_{n+1}(x)=Q_{n+1}(x)-\varepsilon(n+1) Q_{n}(x), n \geqslant 0, \\
& P_{n+1}^{\prime}(x)=(n+1) Q_{n}(x), n \geqslant 0,
\end{aligned}
$$

where $\left\{\beta_{n}\right\}_{n \geqslant 0},\left\{\chi_{n}\right\}_{n \geqslant 0},\left\{\gamma_{n}\right\}_{n \geqslant 0}$ and $\left\{\theta_{n}\right\}_{n \geqslant 0}$ satisfy

$$
\begin{aligned}
\chi_{n} & =\beta_{n}-\varepsilon, n \geqslant 0, \\
\theta_{n+1} & =\gamma_{n+1}+\varepsilon^{2}(n+1), n \geqslant 0 .
\end{aligned}
$$

Proof. By starting (16), with $n$ replaced by $n+2$, and using (17) and (18), we obtain

$\left(x-\chi_{n+1}\right) Q_{n+1}(x)-\theta_{n+1} Q_{n}(x)=$

Equivalently,

$$
=\left(x-\beta_{n+1}\right) Q_{n+1}(x)-\gamma_{n+1} Q_{n}(x)+\varepsilon P_{n+1}(x), n \geqslant 0 .
$$

$$
\left(\beta_{n+1}-\chi_{n+1}\right) Q_{n+1}(x)+\left(\gamma_{n+1}-\theta_{n+1}\right) Q_{n}(x)=\varepsilon P_{n+1}(x), n \geqslant 0 .
$$


By comparing the degrees in the last equation, we obtain $\chi_{n+1}=\beta_{n+1}-\varepsilon, n \geqslant 0$ and then

$$
\varepsilon Q_{n+1}(x)+\left(\gamma_{n+1}-\theta_{n+1}\right) Q_{n}(x)=\varepsilon P_{n+1}(x), n \geqslant 0 .
$$

Making $n=1$ in (16), we get $\chi_{0}=\beta_{0}-\varepsilon$; then (21) is valid. Inserting (16), with $n$ replaced by $n+1$, in (23) we obtain

$$
\varepsilon^{2} P_{n+1}^{\prime}(x)=\left(\theta_{n+1}-\gamma_{n+1}\right) Q_{n}(x), n \geqslant 0 .
$$

After analysis of the degree, we obtain (22). Hence, (19) and (20) are valid.

Based on Lemma 1 and the SSR of Laguerre polynomials, we can state that the scaled Laguerre polynomial sequence $\left\{a^{-n} L_{n}^{(\alpha)}(a x)\right\}_{n \geqslant 0}$ where $a=-\varepsilon^{-1}$, is the only $\mathfrak{O}_{\varepsilon}$-classical orthogonal sequence. More precisely, for all $n \geqslant 0$,

$$
P_{n}(x)=(-\varepsilon)^{n} L_{n}^{(\alpha)}\left(-\varepsilon^{-1} x\right) \quad \text { and } \quad Q_{n}(x)=(-\varepsilon)^{n} L_{n}^{(\alpha+1)}\left(-\varepsilon^{-1} x\right) .
$$

Theorem 2. For any nonzero complex number $\varepsilon$ and any monic polynomial sequence $\left\{P_{n}\right\}_{n \geqslant 0}$, the following statements are equivalent.

(i) $\left\{P_{n}\right\}_{n \geqslant 0}$ is an $\mathfrak{O}_{\varepsilon}$-classical orthogonal sequence.

(ii) There exists $a \in \mathbb{C}, a \neq 0$ such that $P_{n}(x)=a^{-n} L_{n}^{(\alpha)}(a x), n \geqslant 0$.

Proof. (i) $\Rightarrow$ (ii). Assume that $\left\{P_{n}\right\}_{n \geqslant 0}$ is a monic $\mathfrak{O}_{\varepsilon}$-classical orthogonal sequence. Then, there exists a monic orthogonal sequence $\left\{Q_{n}\right\}_{n \geqslant 0}$ that satisfies (16) and gives, after inserting in (19),

$$
P_{n}(x)=\frac{1}{n+1} P_{n+1}^{\prime}(x)-\varepsilon P_{n}^{\prime}(x), n \geqslant 0 .
$$

Essentially, (24) corresponds to the scaled Laguerre polynomial sequence

$$
\left\{(-\varepsilon)^{n} L_{n}^{(\alpha)}\left(-\varepsilon^{-1} x\right)\right\}_{n \geqslant 0},
$$

(see (12)), i. e., $P_{n}(x)=(-\varepsilon)^{n} L_{n}^{(\alpha)}\left(-\varepsilon^{-1} x\right), n \geqslant 0,(\alpha \neq-n, n \geqslant 1)$, where, from (10) and (7), we have

$\beta_{n}=-\varepsilon(2 n+\alpha+1), n \geqslant 0 \quad$ and $\quad \gamma_{n+1}=\varepsilon^{2}(n+1)(n+\alpha+1), n \geqslant 0$.

In the same way, from (21) and (22), we obtain

$$
\chi_{n}=-\varepsilon(2 n+\alpha+2), n \geqslant 0, \quad \theta_{n+1}=\varepsilon^{2}(n+1)(n+\alpha+2), n \geqslant 0 .
$$


Then, we also conclude that $Q_{n}(x)=(-\varepsilon)^{n} L_{n}^{(\alpha+1)}\left(-\varepsilon^{-1} x\right), n \geqslant 0$.

(ii) $\Rightarrow$ (i). Let $a$ in $\mathbb{C}$, with $a \neq 0$ and let $P_{n}(x)=a^{-n} L_{n}^{(\alpha)}(a x), n \geqslant 0$. It is clear that $\left\{P_{n}\right\}_{n \geqslant 0}$ is a MOPS. By using the the (SSR) (12) satisfied by $L_{n}^{(\alpha)}(x), n \geqslant 0$ and the relation (1), we have

$$
L_{n}^{(\alpha+1)}(x)=L_{n}^{(\alpha)}(x)-L_{n}^{(\alpha)^{\prime}}(x), \quad n \geqslant 0 .
$$

Besides, from (25), where $x$ is replaced by $a x$, it comes that

$$
L_{n}^{(\alpha+1)}(a x)=L_{n}^{(\alpha)}(a x)-a^{-1}\left(L_{n}^{(\alpha)}(a x)\right)^{\prime}, \quad n \geqslant 0,
$$

or, equivalently, $a^{-n} L_{n}^{(\alpha+1)}(a x)=\left(\mathbb{I}-a^{-1} D\right) a^{-n} L_{n}^{(\alpha)}(a x), n \geqslant 0$, i. e.,

$$
\mathfrak{O}_{\varepsilon} P_{n}(x)=a^{-n} L_{n}^{(\alpha+1)}(a x), \quad n \geqslant 0,
$$

where $\varepsilon=-a^{-1}$. Hence, (i) holds, since $\left\{a^{-n} L_{n}^{(\alpha+1)}(a x)\right\}_{n \geqslant 0}$ is a MOPS.

4. Higher-order differential relations. As a consequence of Section 3, we have

$$
\mathfrak{O}_{\varepsilon} L_{n}^{(\alpha)}\left(-\varepsilon^{-1} x\right)=L_{n}^{(\alpha+1)}\left(-\varepsilon^{-1} x\right), n \geqslant 0 .
$$

If we take $\varepsilon=-1$ and $\mathfrak{O}_{-1}:=\mathfrak{O}$, we have the canonical situation

$$
\mathfrak{O} L_{n}^{(\alpha)}(x)=L_{n}^{(\alpha+1)}(x), n \geqslant 0,
$$

which gives, by induction on $m \in \mathbb{N}$,

$$
\mathfrak{O}^{m} L_{n}^{(\alpha)}(x)=L_{n}^{(\alpha+m)}(x), n \geqslant 0, \quad\left(\mathfrak{D}^{0}=\mathbb{I}\right) .
$$

Note that by using (1), the polynomial $L_{n}^{(\alpha+m)}(x)$ can be written as follows

$$
\begin{aligned}
L_{n}^{(\alpha+m)}(x) & =\frac{1}{(n+1)(n+2) \cdots(n+m)} D^{m} L_{n+m}^{(\alpha)}(x), n \geqslant 0, m \geqslant 0, \\
& =\frac{n !}{(n+m) !} D^{m} L_{n+m}^{(\alpha)}(x), n \geqslant 0, m \geqslant 0
\end{aligned}
$$

and, then, we get the following relation between $\mathfrak{O}^{m}$ and $D^{m}$ :

$$
\mathfrak{O}^{m} L_{n}^{(\alpha)}(x)=\frac{n !}{(n+m) !} D^{m} L_{n+m}^{(\alpha)}(x), n \geqslant 0, m \geqslant 0,
$$


with the convention $\mathfrak{O}^{0}=D^{0}=\mathbb{I}$.

By (26), with $\alpha=0$, and using the fact that $\mathfrak{O}^{m}=(\mathbb{I}-D)^{m}$ and the binomial formula, we can state the following result.

Lemma 2. The monic Laguerre polynomials $L_{n}^{(m)}(x), m \geqslant 0$, are represented in terms of the action of linear differential operators on the Laguerre polynomials $L_{n}^{(0)}(x)$, as follows:

$$
L_{n}^{(m)}(x)=\sum_{\nu=0}^{m}(-1)^{\nu}\left(\begin{array}{c}
m \\
\nu
\end{array}\right) D^{\nu} L_{n}^{(0)}(x), n \geqslant 0 .
$$

Having Lemma 2, it is natural to study if the reciprocal is true. Firstly, we need the following relation, obtained from the explicit expression for the Laguerre polynomials

$$
x L_{n}^{(1)^{\prime}}(x)+L_{n}^{(1)}(x)=(n+1) L_{n}^{(0)}(x), \quad n \geqslant 1 .
$$

Theorem 3. The representation of the Laguerre polynomials $L_{n}^{(0)}(x)$ in terms of action of linear differential operators on the Laguerre polynomials $L_{n}^{(m)}(x)$, is given by

$$
L_{n}^{(0)}(x)=\frac{n !}{(n+m) !} \sum_{\nu=0}^{m}\left[\left(\begin{array}{c}
m \\
\nu
\end{array}\right)\right]^{2} \nu ! x^{m-\nu} D^{m-\nu} L_{n}^{(m)}(x), n \geqslant 0 .
$$

Proof. We prove this by induction on $m \in \mathbb{N}$. For $m=0$ this is obvious. Now suppose (28) holds and prove the same for $m+1$ instead of $m$. Indeed, by differentiating both sides of (28) and using (1), with $\alpha=0$, we get, for all $n \geqslant 1$,

$$
\begin{aligned}
\frac{n !}{(n+m) !} \sum_{\nu=0}^{m}\left[\left(\begin{array}{c}
m \\
\nu
\end{array}\right)\right]^{2} & \nu !\left(x^{m-\nu} D^{m-\nu}+\right. \\
& \left.+(m-\nu) x^{m-\nu-1} D^{m-\nu-1}\right) L_{n-1}^{(m+1)}(x)=L_{n-1}^{(1)}(x) .
\end{aligned}
$$

Multiplying both sides of the previous equation by $x$, applying the operator $D$, and using the identity (27), we obtain for all $n \geqslant 1$

$$
\frac{(n-1) !}{(n+m) !}\left\{\sum_{\nu=0}^{m} \frac{(m !)^{2}}{[(m-\nu) !]^{2} \nu !} x^{m+1-\nu} D^{m+1-\nu}+\right.
$$




$$
\begin{gathered}
+\sum_{\nu=0}^{m} \frac{(2 m-2 \nu+1)(m !)^{2}}{[(m-\nu) !]^{2} \nu !} x^{m-\nu} D^{m-\nu}+ \\
\left.+\sum_{\nu=0}^{m-1} \frac{(m !)^{2}}{[(m-1-\nu) !]^{2} \nu !} x^{m-1-\nu} D^{m-1-\nu}\right\} L_{n-1}^{(m+1)}(x)=L_{n-1}^{(0)}(x) .
\end{gathered}
$$

By replacing $\nu$ by $\nu-1$ (resp. $\nu-2$ ) in the second (resp. third) sum, we obtain for all $n \geqslant 1$

$$
\begin{gathered}
\frac{(n-1) !}{(n+m) !} \sum_{\nu=2}^{m}\left\{\frac{(m !)^{2}}{[(m-\nu) !]^{2} \nu !}+\frac{(2 m-2 \nu+3)(m !)^{2}}{[(m+1-\nu) !]^{2}(\nu-1) !}+\right. \\
\left.+\frac{(m !)^{2}}{[(m+1-\nu) !]^{2}(\nu-2) !}\right\} x^{m+1-\nu} D^{m+1-\nu} L_{n-1}^{(m+1)}(x)+ \\
+\frac{(n-1) !}{(n+m) !}\left(x^{m+1} D^{m+1}+(m+1)^{2} x^{m} D^{m}+(m+1) ! \mathbb{I}\right) L_{n-1}^{(m+1)}(x)=L_{n-1}^{(0)}(x) .
\end{gathered}
$$

After some calculations, with $n$ replaced by $n+1$, we finally obtain for all $n \geqslant 0$

$$
L_{n}^{(0)}(x)=\frac{n !}{(n+m+1) !} \sum_{\nu=0}^{m+1}\left[\left(\begin{array}{c}
m+1 \\
\nu
\end{array}\right)\right]^{2} \nu ! x^{m+1-\nu} D^{m+1-\nu} L_{n}^{(m+1)}(x) .
$$

Hence the desired result is proved.

4. Integral formulas. Consider the integral operator [14]:

$$
\mathfrak{S}_{c}(P)(x)=\int_{0}^{+\infty} t e^{-t} P(t(x-c)+c) \mathrm{d} t, \quad c \in \mathbb{C}, P \in \mathcal{P} .
$$

In particular, for $P(x)=(x-c)^{n}$, we have

$$
\mathfrak{S}_{c}\left((x-c)^{n}\right)=(n+1) !(x-c)^{n}, \quad n \geqslant 0 .
$$

By (30) and (13), it is easily seen that for every integer $m \in \mathbb{N} \backslash\{0\}$

$$
\mathfrak{S}_{0}\left(x^{m-1} L_{n}^{(m)}(x)\right)=(n+m) ! x^{m-1}(x-1)^{n}, \quad n \geqslant 0 .
$$

Equivalently,

$$
x^{n}=\frac{1}{(n+m) !} \int_{0}^{+\infty} t^{m} e^{-t} L_{n}^{(m)}(t(x+1)) \mathrm{d} t, \quad n \geqslant 0 .
$$


Now, as an application of (31), some connection formulas between the monomial basis $\left\{x^{n}\right\}_{n \geqslant 0}$ and the shifted Laguerre basis $\left\{L_{n}^{(m)}(x+1)\right\}_{n \geqslant 0}$ are deduced.

\section{Theorem 4.}

(i) For every integer $m \geqslant 1$, the following formulas hold for all $n \geqslant 0$

$$
\begin{gathered}
x^{n}=\sum_{k=0}^{n}\left(\begin{array}{l}
n \\
k
\end{array}\right) \frac{(-1)^{n-k}}{(m+k) !} L_{k}^{(m)}(x+1) \int_{0}^{+\infty} t^{m+k}(1-t)^{n-k} e^{-t} \mathrm{~d} t, \\
x^{n}=\sum_{k=0}^{n} \sum_{i=0}^{n-k}\left(\begin{array}{l}
n \\
k
\end{array}\right)\left(\begin{array}{c}
n-k \\
i
\end{array}\right) \frac{(-1)^{k}(m+k+i) !}{(m+k) !} L_{k}^{(m)}(x+1) .
\end{gathered}
$$

(ii) For $m=0$, we have for all $n \geqslant 0$

$$
x^{n}=\sum_{k=0}^{n} \frac{(-1)^{k}}{k !} \frac{(-1)^{n}}{n+1} \int_{0}^{+\infty} t e^{-t} L_{k}^{(0)}(t(x+1)) \mathrm{d} t .
$$

Proof. By inserting (14), with $\alpha$ replaced by $m$ and $x$ by $x+1$, in (31), we obtain

$$
x^{n}=\sum_{k=0}^{n}\left(\begin{array}{l}
n \\
k
\end{array}\right) \frac{(-1)^{n-k}}{(m+k) !} L_{k}^{(m)}(x+1) \int_{0}^{+\infty} t^{m+k}(1-t)^{n-k} e^{-t} \mathrm{~d} t, \quad n \geqslant 0 .
$$

Then (32) follows.

Substitute the binomial formula for $(1-t)^{n-k}$, in this last equality, obtaining

$$
\begin{aligned}
x^{n} & =\sum_{k=0}^{n}\left(\begin{array}{l}
n \\
k
\end{array}\right) \frac{(-1)^{n-k}}{(m+k) !} L_{k}^{(m)}(x+1) \sum_{i=0}^{n-k}(-1)^{n}\left(\begin{array}{c}
n-k \\
i
\end{array}\right)(m+k+i) != \\
& =\sum_{k=0}^{n} \sum_{i=0}^{n-k}\left(\begin{array}{l}
n \\
k
\end{array}\right)\left(\begin{array}{c}
n-k \\
i
\end{array}\right) \frac{(-1)^{k}(m+k+i) !}{(m+k) !} L_{k}^{(m)}(x+1) .
\end{aligned}
$$

Hence we obtain (33).

For (ii), using (30) and (13) with $\alpha=m=0$, the operator $\mathfrak{S}_{0}$ satisfies 


$$
\begin{gathered}
\mathfrak{S}_{0}\left(L_{n}^{(0)}(x)\right)(x)=n ! \sum_{\nu=0}^{n}(-1)^{n-\nu}\left(\begin{array}{l}
n \\
\nu
\end{array}\right)(\nu+1) x^{\nu}= \\
=n !\left[\sum_{\nu=1}^{n}(-1)^{n-\nu} \frac{n !}{(n-\nu) !(\nu-1) !} x^{\nu}+\sum_{\nu=0}^{n}(-1)^{n-\nu}\left(\begin{array}{l}
n \\
\nu
\end{array}\right) x^{\nu}\right]= \\
=n !\left[n x \sum_{\nu=0}^{n-1}(-1)^{n-\nu-1}\left(\begin{array}{c}
n-1 \\
\nu
\end{array}\right) x^{\nu}+(x-1)^{n}\right]= \\
=n !(x-1)^{n-1}[(n+1) x-1], \quad n \geqslant 1 .
\end{gathered}
$$

Using (34), with $x$ replaced by $x+1$, we obtain the following integral relation

$$
(n+1) x^{n}+n x^{n-1}=\frac{1}{n !} \int_{0}^{+\infty} t e^{-t} L_{n}^{(0)}(t(x+1)) \mathrm{d} t, \quad n \geqslant 1 .
$$

This gives, by summation, the following result for all $n \geqslant 1$

$$
\sum_{k=1}^{n}\left[(k+1)(-x)^{k}-k(-x)^{k-1}\right]=\int_{0}^{+\infty} t e^{-t} \sum_{k=1}^{n} \frac{(-1)^{k}}{k !} L_{k}^{(0)}(t(x+1)) \mathrm{d} t .
$$

Taking a telescopic sum, we get

$$
(n+1)(-x)^{n}-1=\int_{0}^{+\infty} t e^{-t} \sum_{k=1}^{n} \frac{(-1)^{k}}{k !} L_{k}^{(0)}(t(x+1)) \mathrm{d} t, \quad n \geqslant 1 .
$$

Thus, the basic $\left\{x^{n}\right\}_{n \geqslant 0}$ satisfies

$$
x^{n}=\frac{(-1)^{n}}{n+1} \int_{0}^{+\infty} t e^{-t} \sum_{k=0}^{n} \frac{(-1)^{k}}{k !} L_{k}^{(0)}(t(x+1)) \mathrm{d} t, \quad n \geqslant 0 .
$$

Hence, the desired result is proved.

Remark 1. By substituting (15) into (31), with $\alpha$ replaced by $m$ and $x$ by $x+1$, we obtain for all $n \geqslant 0$

$$
x^{n}=\frac{n ! m ! 4^{m}}{(2 n) !(2 m) ! \pi} \int_{-1}^{1} \int_{0}^{+\infty}\left(1-y^{2}\right)^{m-\frac{1}{2}} t^{m} e^{-t} H_{2 n}(y \sqrt{t(x+1)}) \mathrm{d} t \mathrm{~d} y,
$$


which gives, for $n=0$,

$$
\begin{gathered}
1=\frac{m ! 4^{m}}{(2 m) ! \pi} \int_{-1}^{1}\left(1-y^{2}\right)^{m-\frac{1}{2}} \mathrm{~d} y \int_{0}^{+\infty} t^{m} e^{-t} \mathrm{~d} t= \\
\quad=\frac{(m !)^{2} 4^{m}}{(2 m) ! \pi} \int_{-1}^{1}\left(1-y^{2}\right)^{m-\frac{1}{2}} \mathrm{~d} y .
\end{gathered}
$$

Then, if we pose $y=\sin \theta$ in (35), we recover the Wallis integral

$$
\int_{0}^{\frac{\pi}{2}} \sin ^{2 m} \theta \mathrm{d} \theta=\frac{(2 m) ! \pi}{2^{2 m+1}(m !)^{2}}, m \geqslant 0 .
$$

Acknowledgment. The authors are very grateful to the referees for the constructive comments and for making us pay attention to a certain reference.

\section{References}

[1] Aloui B., Marcellán F., Sfaxi R. Classical orthogonal polynomials with respect to a lowering operator generalizing the Laguerre operator. Integral Transforms Spec. Funct., 2013, no. 24, pp. 636-648.

DOI: https://doi.org/10.1080/10652469.2012.744006

[2] Aloui B. Characterization of Laguerre polynomials as orthogonal polynomials connected by the Laguerre degree raising shift operator. Ramanujan J., 2018, no. 45, pp. $475-481$.

DOI: https://doi.org/10.1007/s11139-017-9901-x

[3] Aloui B. Chebyshev polynomials of the second kind via raising operator preserving the orthogonality. Period. Math. Hung., 2018, no. 76, 126-132. DOI: https://doi.org/10.1007/s10998-017-0219-7

[4] Aloui B. Hermite polynomials and Hahn's theorem with respect to the raising operator. Electr. J. Math. Anal. Appl., 2018, vol. 6 (2), pp. 157-162. DOI: http://fcag-egypt.com/Journals/EJMAA/

[5] Al-Salam W. A. Characterization theorems for orthogonal polynomials. in: Orthogonal polynomials: theory and practice. NATO ASI Series C, vol. 294, Kluwer, 1990, pp. 1-24. 
[6] Area I., Godoy A., Ronveaux A., Zarzo A. Classical symmetric orthogonal polynomials of a discrete variable. Integral Transforms Spec. Funct., 2004, vol. 15 , pp. $1-12$.

DOI: https://doi.org/10.1080/10652460310001600672

[7] Askey R. Divided difference operators and classical orthogonal polynomials. Rocky Mountain J. Math., 1989, vol. 19, pp. 33-37.

[8] Ben Cheikh Y., Gaied M. Characterization of the Dunkl-classical symmetric orthogonal polynomials. Appl. Math. Comput., 2007, vol. 187, pp. $105-$ 114. DOI: https://doi.org/10.1016/j.amc.2006.08.108

[9] Bochner S. Über Sturm-Liouvillesche Polynomsysteme. Z. Math., 1929, vol. 29, pp. $730-736$.

[10] Chihara T. S. An Introduction to Orthogonal Polynomials. Gordon and Breach, New York, 1978.

[11] Dattoli G., Ricci P. E. Laguerre-type exponentials, and the relevant Lcircular and L-hyperbolic functions. Georgian Math. J., 2003, vol. 10, pp. $481-494$.

[12] Hahn W. Über die Jacobischen polynome und zwei verwandte polynomklassen. Math. Z., 1935, vol. 39, pp. 634-638.

[13] Hahn W. Über Orthogonalpolynome, die q-Differenzengleichungen genügen. Math. Nach., 1949, vol. 2, pp. 4-34.

[14] Hochstadt H. The Functions of Mathematical Physics. Dover Publications Inc. New York, 1971.

[15] Khériji L., Maroni P. The $H_{q}$-classical orthogonal polynomials. Acta. Appl. Math., 2002, vol. 71, pp. 49-115.

DOI: https://doi.org/10.1023/A:1014597619994

[16] Koekoek R and Swarttouw R. F. The Askey-scheme of hypergeometric orthogonal polynomials and its q-analogue. Delft University of Technology, Faculty of Information Technology and Systems, Department of Technical Mathematics and Informatics, Report no. 98-17, 1998.

[17] Lebedev N. N. Special functions and their applications. (Revised English Edition, Translated and Edited by Richard Silverman 1965), Dover Publications New York, 1972.

[18] Lesky P. Über polynomsysteme, die Sturm-Liouvilleschen differenzengleichungen genügen. Math. Zeit., 1962, vol. 78, pp. 439-445.

[19] Loureiro A. F., Maroni P. Quadratic decomposition of Appell sequences. Expo. Math., 2008, vol. 26, pp. $177-186$.

DOI: https://doi.org/10.1016/j.exmath.2007.10.002 
[20] Maroni P. Fonctions Eulériennes, Polynômes Orthogonaux Classiques. Techniques de l'Ingénieur, Traité Généralités (Sciences Fondamentales). A 154, 1-30 Paris, 1994.

[21] Maroni P. Une théorie algébrique des polynômes orthogonaux. Applications aux polynômes orthogonaux semi-classiques. In: Orthogonal Polynomials and their Applications, C. Brezinski et al. Editors, IMACS Ann. Comput. Appl. Math., 1991, no. 9, pp. 95-130.

[22] Maroni P. Variations autour des polynômes orthogonaux classiques. C. R. Acad. Sci. Paris Sér. I Math., 1991, vol. 313, pp. 209-212.

[23] Nikoforov A. F, Ouvarov V. B. Special Functions of Mathematical Physics. Birkhäuser, Basel, Boston, 1988.

[24] Sonine N. J. On the approximate computation of definite integrals and on the entire functions occurring there. Warsch. Univ. Izv., 1887, vol. 18, pp. $1-76$.

[25] Szegö G. Orthogonal Polynomials. Amer. Math. Soc. Colloq. Publ., Vol. 23, Amer. Math. Soc., Providence, RI, 1975.

[26] Tincu I. Some properties from Laguerre polynomials. Gen. Math., 2010, vol. 18 , no. 1 , pp. $131-137$.

Received May 14, 2019.

In revised form, September 23, 2019.

Accepted October 01, 2019.

Published online October 09, 2019.

B. Aloui

Université de Gabès, Institut Supérieur des Systèmes Industriels de Gabès,

Rue Salah Eddine Elayoubi 6033 Gabès, Tunisia.

E-mail: baghdadi.aloui@fsg.rnu.tn

L. Khériji

Université de Tunis El Manar, Institut Préparatoire aux Etudes d'Ingénieur El Manar, Campus Universitaire El Manar, B.P. 244, 2092 Tunis, Tunisia.

LR13ES06.

E-mail: kheriji@yahoo.fr or Lotfi.Kheriji@issatgb.rnu.tn 